\title{
Laparoscopic Observations in Acquired Inguinal Hernia before and after Robotic-Assisted Laparoscopic Prostatectomy
}

\author{
Masahiko Kawaguchi', Yoshifumi Kadono², Masanari Shimada1, Hideki Moriyama1, \\ Norihiko Ishikawa1, Go Watanabe ${ }^{1}$ \\ ${ }^{1}$ Departments of General and Cardiothoracic Surgery, Kanazawa University, Kanazawa, Japan \\ ${ }^{2}$ Departments of Urology, Kanazawa University, Kanazawa, Japan \\ Email: surgkw@gmail.com
}

Received 11 March 2014; revised 5 April 2014; accepted 12 April 2014

Copyright (C) 2014 by authors and Scientific Research Publishing Inc.

This work is licensed under the Creative Commons Attribution International License (CC BY). http://creativecommons.org/licenses/by/4.0/

(c) (i) Open Access

\section{Abstract}

Background: The mechanism of the development of acquired inguinal hernia, especially indirect inguinal hernia, is not well known. Although anatomical studies have been performed to explain development of inguinal hernia, they have mainly involved autopsy or temporal findings at the time of hernioplasty. To elucidate the pattern of development of acquired inguinal hernia, we studied the occurrence of inguinal hernia after robotic-assisted laparoscopic prostatectomy (RALP). Methods: From March 2009 to November 2011, RALP for prostatic cancer was performed on 60 patients in our institute. The RALP was performed by one urologist using the da Vinci Surgical System. The postsurgical development of inguinal hernia was diagnosed based on patients' symptoms. Seven patients were treated with laparoscopic hernioplasty, and one underwent mesh-plug repair. Using video recordings, the laparoscopic findings during RALP and laparoscopic hernioplasty were compared among all patients. Results: Seven of 59 patients $(11.9 \%)$ developed an inguinal hernia. One patient had a pre-existing pantaloon inguinal hernia at the time of RALP. Eleven inguinal lesions in the seven patients who underwent laparoscopic hernioplasty were reviewed, and all were indirect inguinal hernias. Conclusion: A main factor in the development of inguinal hernia after RALP could be a combination of outer-side intact layers and inner-side hard scar of the inguinal ring, which seems like "out swing door".

\section{Keywords}

Acquired Inguinal Hernia, Laparoscopic Hernioplasty, Robotic-Assisted Laparoscopic Prostatectomy

How to cite this paper: Kawaguchi, M., Kadono, Y., Shimada, M., Moriyama, H., Ishikawa, N. and Watanabe, G. (2014) Laparoscopic Observations in Acquired Inguinal Hernia before and after Robotic-Assisted Laparoscopic Prostatectomy. Surgical Science, 5, 170-175. http://dx.doi.org/10.4236/ss.2014.54030 


\section{Introduction}

Acquired inguinal hernia is common. However, the mechanism of its development, especially indirect inguinal hernia, is not well known. One reason for this is the difficulty in defining the etiology. The factors associated with the development of inguinal hernia are considered to be an increase in abdominal pressure with dehiscence of the abdominal wall, which may be related to injury, or attenuation of the inguinal wall [1].

The causes of acquired hernia are physiological actions or exercises such as coughing and straining. Moreover, external pressure, some surgical procedures, and even geriatric attenuation may be additional causes. However, the cause of most acquired inguinal hernias is undefined because most are subjectively detected by patients' symptoms and may develop long time after the cause. From an anatomical aspect, injury to or attenuation of the posterior wall of the inguinal canal, the presence of a patent processus vaginalis (PPV), failure of the shutter mechanism, and dilatation of the inguinal ring have been described as factors associated with the development of inguinal hernias [2]. Many anatomical studies have been performed using autopsy and intraoperative surgical findings. However, the findings in these studies are temporal; studies revealing the direct pre-development and post-development findings of inguinal hernia are lacking.

Among the causes of acquired inguinal hernia, prostatectomy is one of the most studied [3]. The etiology has been defined, and postprostatectomy inguinal hernia is considered to be an aggravating complication. The anatomical factors of inguinal hernia after prostatectomy have been described as PPV and injury to the posterior rectal sheath [3]. However, the factors that may contribute to the development of inguinal hernia after robotic-assisted laparoscopic prostatectomy (RALP) are still unclear.

Therefore, we studied the development of inguinal hernia after RALP by chronological video-recorded inguinal observations at the time of RALP and laparoscopic hernioplasty. Comparing our observations before and after the development inguinal hernia, we considered the mechanisms of and factors associated with acquired inguinal hernia.

\section{Patients and Methods}

From March 2009 to November 2011, RALP for prostatic cancer was performed on 60 patients in our institute. The RALP was performed by one urologist using the da Vinci Surgical System (Intuitive Surgical Inc., Sunnyvale, CA, USA). During the procedure, three ports were added by an assistant surgeon. After the robotic settings, the peritoneal incision was started between the lateral umbilical fold and inferior epigastric vessels, and peritoneal dissection continued toward the midline. Therefore, the operative field during the RALP did not invade the internal inguinal ring.

The patients' clinicopathological data are shown in Table 1. Data on inguinal hernias after RALP, which were detected by symptoms such as inguinal swelling or pain, were gathered without concern about the presence of an inguinal hernia before RALP. Most symptoms were inguinal swelling and pain. The inguinal hernia development times after RALP were also evaluated in all patients with the exception of one, who had already an inguinal hernia before RALP.

Our procedure for inguinal hernia repair was mainly laparoscopic hernioplasty using a modified intraperitonealonlay method [4] with C-QUR Edge (Atrium Medical Corporation, Hudson, NH, USA); specifically, transabdominal preperitoneal patch repair without peritoneal reconstruction. Open methods were performed using mesh and plugs.

The inguinal regions were retrospectively reviewed using videos during RALP and laparoscopic hernioplasty. On the RALP videos, the presence of a processus vaginalis or inguinal hernia was observed if the inguinal regions were recorded in the video. Surgical interventions involving the inguinal posterior wall were also observed.

On the laparoscopic hernioplasty videos, the inguinal region, which included the presence, type, and location of the inguinal hernia, was observed. A comparison of the inguinal regions was then performed (Figure 1, Figure 2). All hernias were classified according to the European Hernia Society classification [5].

Statistical analysis was performed using R 2.12 software. Numerical data were given as median and range, and categorical variables were expressed by number (n). Continuous variables were compared using the Student's t-test, and frequency analysis was performed with Fisher's exact test. Differences were assessed with twosided tests, with an alpha level of 0.05 . 
Table 1. Comparison of development of inguinal hernia after robotic-assisted laparoscopic prostatectomy.

\begin{tabular}{cccc}
\hline & No inguinal hernia & Inguinal hernia & $p$-value \\
\hline Number of cases & 52 & 8 & 0.946 \\
Age & 65.0 & 65.1 & 0.670 \\
Height (cm) & 164.4 & 165.2 & 0.187 \\
Weight (kg) & 65.5 & 63.8 & 0.230 \\
Body mass index & 26.4 & 23.4 & 1 \\
Past operation for inguinal hernia & 3 & 0 & 0.0014 \\
Clinical stage of prostatic cancer & T1: 30 & T1: & 1 \\
Hormonal therapy (yes:no) & T3: 20 & T3: $: 3$ & 0.187 \\
Operating time (min) & $29: 23$ & $3: 5$ & 0.230 \\
Console time (min) & 228.7 & 202.9 & 0.883 \\
Blood loss (ml) & 176.4 & 155.2 & 297.5 \\
\hline
\end{tabular}

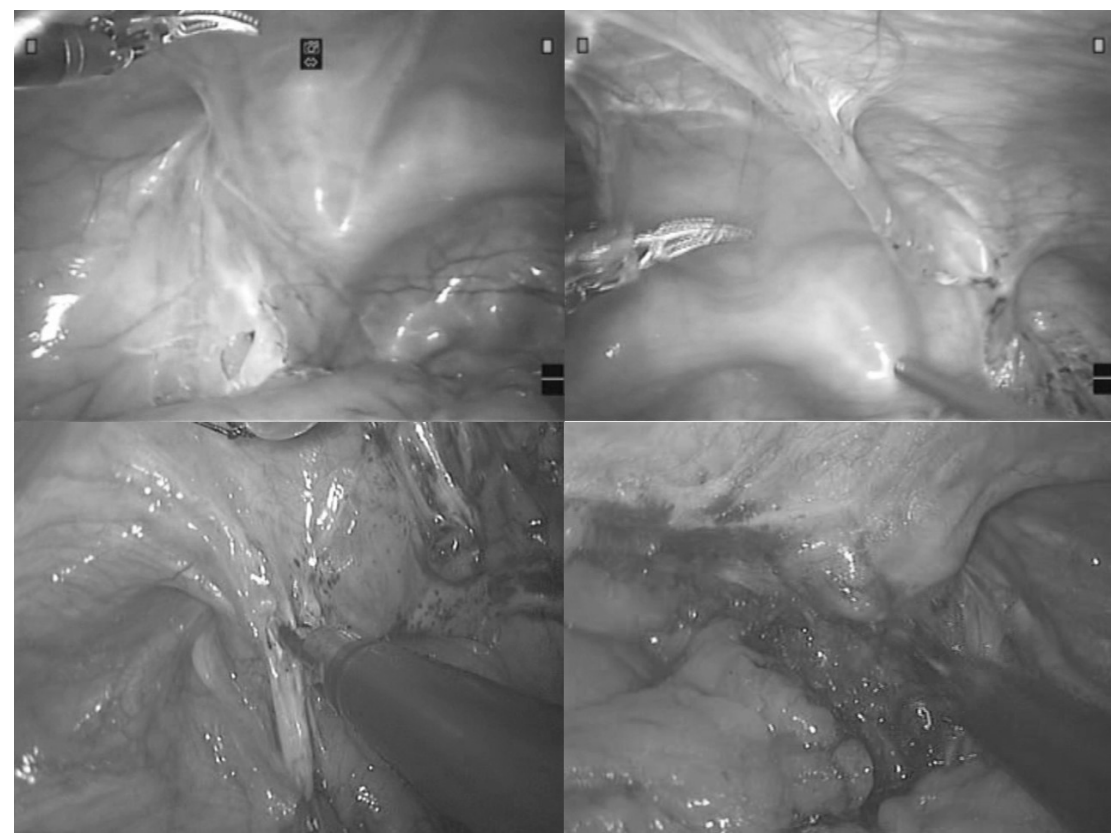

Figure 1. Internal observations of the bilateral inguinal region: comparison between robotic-assisted laparoscopic prostatectomy (RALP) and laparoscopic hernioplasty. The upper pictures from RALP show a bilateral remaining processus vaginalis. The lower pictures from laparoscopic hernioplasty show postoperative inguinal hernias, which are similar in shape to the preoperative processus vaginalis

\section{Results}

Sixty patients were evaluated during the study period (Table 1). Seven patients $(7 / 59,11.9 \%)$ developed an inguinal hernia. One patient had a pantaloon inguinal hernia and underwent RALP without hernioplasty for economic reasons. The eight patients who had inguinal hernias after RALP were reviewed by RALP surgical videos, and seven of them underwent laparoscopic hernioplasty (Table 2). Eleven inguinal lesions in the seven patients who underwent laparoscopic hernioplasty were reviewed by videos of laparoscopic hernioplasty, and all lesions were indirect inguinal hernias. Only clinical stage showed statistical significance in terms of inguinal hernia development between patients who did and did not develop an inguinal hernia (Table 1). 


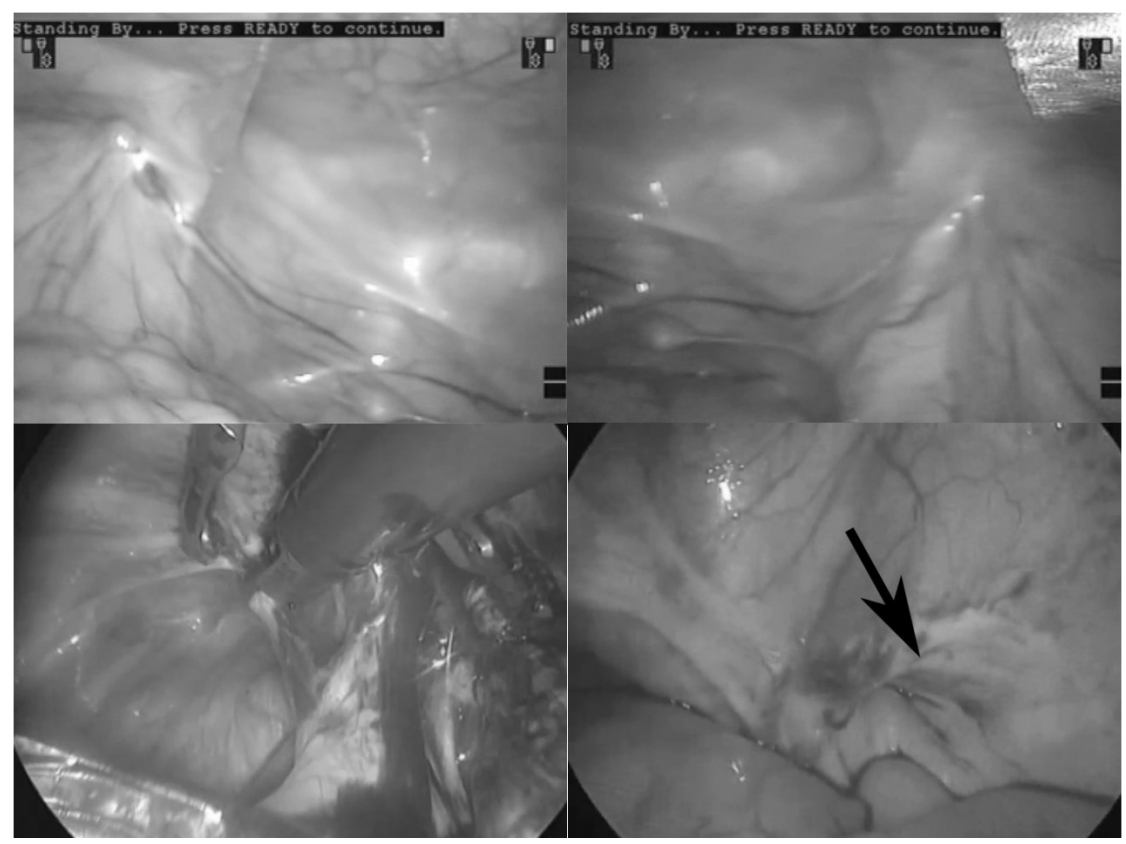

Figure 2. Internal observations of the bilateral inguinal region: comparison between robotic-assisted laparoscopic prostatectomy (RALP) and laparoscopic hernioplasty. The upper pictures from RALP show a small processus vaginalis on the left and lack of a processus vaginalis on the right. The lower pictures from laparoscopic hernioplasty show bilaterally developing inguinal hernias. The arrow shows a cuff scar in the neck of the inguinal hernia sac.

Table 2. Observations of inguinal hernia after robotic-assisted laparoscopic prostatectomy.

\begin{tabular}{|c|c|c|c|c|c|c|}
\hline Case & Age & $\begin{array}{c}\text { Remaining } \\
\text { processus vaginalis }\end{array}$ & $\begin{array}{l}\text { Duration from } \\
\text { RALP }\end{array}$ & Side & $\begin{array}{c}\text { Classified } \\
\text { by EHS classification }\end{array}$ & Surgery for hernia \\
\hline 1 & 62 & $\begin{array}{l}\text { R: small } \\
\text { L: none }\end{array}$ & 4 months & Right & L2 & Laparoscopy \\
\hline 2 & 59 & $\begin{array}{l}\text { R: none } \\
\text { L: NA }\end{array}$ & 5 months & $\begin{array}{c}\text { Right } \\
\text { Left }\end{array}$ & $\begin{array}{l}\mathrm{L} 2 \\
\mathrm{~L} 2\end{array}$ & Open \\
\hline 3 & 69 & $\begin{array}{l}\text { R: none } \\
\text { L: small }\end{array}$ & 6 months & $\begin{array}{l}\text { Right } \\
\text { Left }\end{array}$ & $\begin{array}{l}\text { L1 } \\
\text { L2 }\end{array}$ & Laparoscopy \\
\hline 4 & 62 & L: NA & 7 months & Left & L2 & Laparoscopy \\
\hline 5 & 65 & $\begin{array}{l}\mathrm{R}: \text { small } \\
\mathrm{L} \text { : small }\end{array}$ & 7 months & $\begin{array}{c}\text { Right } \\
\text { Left }\end{array}$ & $\begin{array}{l}\text { L2 } \\
\text { L1 }\end{array}$ & Laparoscopy \\
\hline 6 & 65 & L: small & 16 months & Left & L2 & Laparoscopy \\
\hline 7 & 76 & $\begin{array}{l}\text { R: small } \\
\text { L: middle }\end{array}$ & 20 months & $\begin{array}{l}\text { Right } \\
\text { Left }\end{array}$ & $\begin{array}{l}\text { L1 } \\
\text { L2 }\end{array}$ & Laparoscopy \\
\hline 8 & 65 & $\begin{array}{l}\text { R: L1M2* } \\
\text { L: middle }\end{array}$ & - & $\begin{array}{c}\text { Right } \\
\text { Left }\end{array}$ & $\begin{array}{l}\mathrm{L} 2 \\
\mathrm{~L} 2\end{array}$ & Laparoscopy \\
\hline
\end{tabular}

RALP indicates robotic-assisted laparoscopic prostatectomy; EHS, European hernia society; R, right side; L, left side; NA, not available; ${ }^{*}$ Preexisted inguinal hernia classified by EHS classification

On the video reviews of the inguinal region, all patients had hard scar formation on the inner side of the inferior epigastric vessels. There was no injuries to the posterior rectus sheath occurred during RALP. Two patterns of inguinal hernia development were observed. First, the presence of a processus vaginalis during RALP was confirmed in eight patients. Similarity of the processus vaginalis and the developed hernia were confirmed by using video images chronologically (Figure 1). In the patients who had a processus vaginalis, less widening of the inguinal ring but deepening of the hernia sac were found. 
On the other hand, at least two patients with newly developed inguinal hernias had no processus vaginalis at RALP. These hernial sacs had a cuff scar at the orifice (Figure 2, arrow), while those scar was not detected in the group with a processus vaginalis.

\section{Discussion}

In the present study, the acquired inguinal hernias were all indirect hernias that had a surgical scar inner side of the hernia. Most of them had PPV previously, however, new development of inguinal hernias without PPV was also detected.

PPV is concerned to be a factor associated with acquired inguinal hernia [6]. In the present study, most acquired hernias were coexistent with PPV. However, asymptomatic PPV does occur [2]. One laparoscopic observational study showed that a PPV was not always congenital, but was acquired [1]. Acquired PPV may result from the tendency to develop an inguinal hernia, such as in cases of inguinal wall attenuation. In any case, the existence of PPV has been proven to be associated with development of acquired inguinal hernia [7]. Therefore, PPV must be a correlation factor of the development of inguinal hernia, however, it could not be defined as a causation.

Another finding of the study was surgical scar adjoining to the inner side of acquired hernia. The relation between the surgical scar and developing inguinal hernia was considered. On the kinetic aspect, a surgical scar by RALP was observed at the inner side of the internal inguinal ring. Thus, the sling of the transversalis fascia was fixed and would not be mobilized [8], which resulted in failure of the shutter mechanism. Therefore, the intraabdominal pressure could make herniation at the inguinal ring.

On the anatomical aspect, abdominal wall of the inguinal region is protected by the transversalis fascia against hernia development [9]. However, the inguinal ring has a gap of the transversalis fascia, because all the layers of the abdominal wall are protruded at the internal inguinal ring [10] [11]. Therefore, the gap must be a vulnerable point at the inguinal region without attenuation of the transversalis fascia.

These arguments raise the consideration about the contents of the gap of the transversalis fascia. The gap is filled with a folding membranous layer, which is the lining layers of the transversalis fascia, such as the peritoneum and preperitoneal membranes. Sometimes, an inguinal lipoma is found among the layers in the gap [12]. Those membranes are thin and each connection among layers is loose, so they are easy to tear and split. Therefore, the inguinal ring must be vulnerable to abdominal pressure and may develop acquired inguinal hernia. In fact, acquired inguinal hernias, which had no PPV previously, were observed a cuff scar at the orifice as a mark of tearing.

According to these considerations, intact layers accompanied with the adjoining surgical scar at the inguinal ring are one of the conditions of developing indirect hernia. The core concept of this is multiple layers with disturbance of shutter mechanism. Therefore, even if some layers were adhered, other layers can be easily split to develop an indirect hernia. Such a development of indirect inguinal hernia seems like an "outswing door".

The present study was retrospective in nature and included a small number of cases. However, meticulous observations and chronological findings of acquired inguinal hernia, as described herein, are scarce. Furthermore, this clearly explanation of acquired indirect inguinal hernia, "outswing door" has never been described.

In the present study, indirect inguinal hernias were developed after RALP. The important factor would be not only the surgical scar at the inner side of the inguinal ring, but also intact layers of the outer side. From this consideration, post prostatectomy state tends to be developed indirect inguinal hernia. Furthermore, prevention of inguinal hernia after prostatectomy has been studied in terms of surgical intervention involving the internal inguinal ring [13]-[15]. Previously described procedures include dissection [13], ligation of the processus vaginalis [14], and figure-8 suturing [15]. Regardless of the kinds of procedures, a common result of these procedures implies fixation of the layers at the inguinal ring. Thus, the concept of "outswing door" can be applied to prevention surgery of such acquired hernia.

Based on the present study, a prospective study to prevent acquired inguinal hernia after RALP is conducted.

\section{References}

[1] Van Wessem, K.J.P., Simons, M.P. and Lange, J.F. (2003) The Etiology of Indirect Inguinal Hernias: Congenital and/or Acquired? Hernia, 7, 76-79. http://dx.doi.org/10.1007/s10029-002-0108-7

[2] Abrahamson, J. (1998) Etiology and Pathophysiology of Primary and Recurrent Groin Hernia Formation. Surgical 
Clinics of North America, 78, 953-972. http://dx.doi.org/10.1016/S0039-6109(05)70364-9

[3] Zhu, S., Zhang, H., Xie, L., Chen, J. and Niu, Y. (2013) Risk Factors and Prevention of Inguinal Hernia after Radical Pros-Tatectomy: A Systematic Review and Meta-Analysis. Journal of Urology, 189, 884-890. http://dx.doi.org/10.1016/j.juro.2012.08.241

[4] Kawaguchi, M., Ishikawa, N., Nishida, Y., Moriyama, H., Kaneki, M., and Watanabe, G. (2012) Laparoscopic Hernioplasty Using Omega-3 Coating Mesh. Surgical Science, 3, 389-392. http://dx.doi.org/10.4236/ss.2012.38077

[5] Miserez, M., Alexandre, J.H., Campanelli, G., Corcione, F., Cuccurullo, D., Pascual, M.H., et al. (2007) The European Hernia Society Groin Hernia Classification: Simple and Easy to Remember. Hernia, 11, 113-116. http://dx.doi.org/10.1007/s10029-007-0198-3

[6] Lee, D.H., Jung, H.B., Chung, M.S. and Chung, B.H. (2013) Patent Processus Vaginalis in Adults Who Underwent Robot-Assisted Laparoscopic Radical Prostatectomy: Predictive Signs of Postoperative Inguinal Hernia in the Internal Inguinal Floor. International Journal of Urology, 20, 177-182. http://dx.doi.org/10.1111/j.1442-2042.2012.03118.x

[7] van Veen, R.N., van Wessem, K.J.P., Halm, J.A., Simons, M.P., Plaisier, P.W., Jeekel, J., et al. (2007) Patent Processus Vaginalis in the Adult as a Risk Factor for the Occurrence of Indirect Inguinal Hernia. Surgical Endoscopy, 21, 202-205. http://dx.doi.org/10.1007/s00464-006-0012-9

[8] Lytle, W.J. (1945) The Internal Inguinal Ring. British Journal of Surgery, 32, 441-446. http://dx.doi.org/10.1002/bjs.18003212802

[9] Mirilas, P., Colborn, G.L., McClusky III, D.A., Skandalakis, L.J., Skandalakis, P.N. and Skandalakis, J.E. (2005) The History of Anatomy and Surgery of the Preperitoneal Space. Archives of Surgery, 140, 90-94. http://dx.doi.org/10.1001/archsurg.140.1.90

[10] Kanaya, S., Tamaki, N., Momoi, H., Gomi, T., Katayama, T., Wada, Y., et al. (2002) Layered Structures of Fascia in the Inguinal Region. Rinsho Geka, 57, 1027-1032.

[11] Sakurai, S. (2001) Tension-Free Hernioplasty. Geka Chiryou, 84, 393-404.

[12] Carilli, S., Alper, A. and Emre, A. (2004) Inguinal Cord Lipomas. Hernia, 8, 252-254.

http://dx.doi.org/10.1007/s10029-004-0231-8

[13] Taguchi, K., Yasui, T., Kubota, H., Fukuta, K., Kobayashi, D., Naruyama, H., et al. (2010) Simple Method of Preventing Postoperative Inguinal Hernia after Radical Retropubic Prostatectomy. Urology, 76, 1083-1087. http://dx.doi.org/10.1016/j.urology.2010.05.002

[14] Fujii, Y., Yamamoto, S., Yonese, J., Kawakami, S., Okubo, Y., Suyama, T., et al. (2010) A Novel Technique to Prevent Postradical Retropubic Prostatectomy Inguinal Hernia: The Processus Vaginalis Transection Method. Urology, 75, 713-717. http://dx.doi.org/10.1016/j.urology.2009.05.051

[15] Stranne, J., Aus, G., Bergdahl, S., Damber, J.-E., Hugosson, J., Khatami, A., et al. (2010) Post-Radical Prostatectomy Inguinal Hernia: A Simple Surgical Intervention Can Substantially Reduce the Incidence-Results from a Prospective Randomized Trial. The Journal of Urology, 184. 984-989. http://dx.doi.org/10.1016/j.juro.2010.04.067 